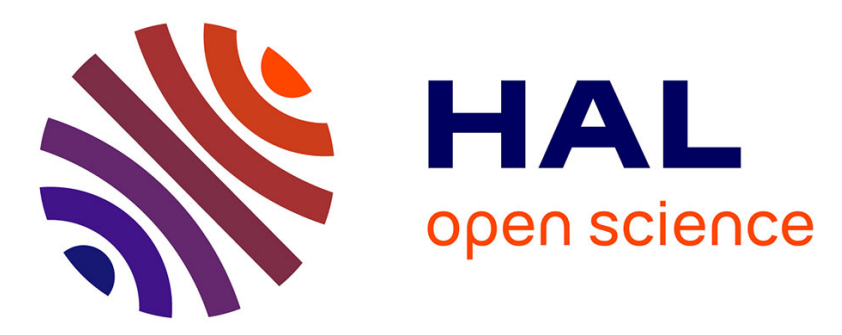

\title{
Stress birefringence patterning in photopolymer induced by structured illumination
}

\author{
Jean-Philippe Bombenger, Alberto Barsella, Christiane Carré, Gregory \\ Taupier, Kokou Dorkenoo, Loic Mager
}

\section{- To cite this version:}

Jean-Philippe Bombenger, Alberto Barsella, Christiane Carré, Gregory Taupier, Kokou Dorkenoo, et al.. Stress birefringence patterning in photopolymer induced by structured illumination. Optical Materials, 2013, 35 (5), pp.923-26. 10.1016/j.optmat.2012.11.008 . hal-00920057

\section{HAL Id: hal-00920057 https://hal.science/hal-00920057}

Submitted on 4 Jul 2014

HAL is a multi-disciplinary open access archive for the deposit and dissemination of scientific research documents, whether they are published or not. The documents may come from teaching and research institutions in France or abroad, or from public or private research centers.
L'archive ouverte pluridisciplinaire HAL, est destinée au dépôt et à la diffusion de documents scientifiques de niveau recherche, publiés ou non, émanant des établissements d'enseignement et de recherche français ou étrangers, des laboratoires publics ou privés. 


\title{
Stress birefringence patterning in photopolymer induced by structured illumination
}

\author{
Jean-Philippe Bombenger ${ }^{\mathrm{a}}$, Alberto Barsella ${ }^{\mathrm{a}}$, Christiane Carréb ${ }^{\mathrm{b}}$, Gregory Taupier ${ }^{\mathrm{a}}$, \\ Kokou Dodzi (Honorat) Dorkenoo ${ }^{a}$, Loïc Mager $^{\text {a }}$ \\ a IPCMS, Groupe d'Optique Non Linéaire et d'Optoélectronique, CNRS UMR 7504, 23 rue du \\ Loess, BP 43, 67034, Strasbourg Cedex 2, France \\ bépartement de Photochimie Générale, CNRS UMR 7525, 3 rue A. Werner, 68093, Mulhouse \\ Cedex, France
}

\begin{abstract}
We report on the stress birefringence distribution induced by the photopatterning of a multifunctional crosslinkable acrylate monomer sensitized in the visible range. The patterning of this material, prepared in a glass cell of a few tens of microns thickness, is obtained through illumination in a two steps process. The first step is performed using an illumination pattern with a high contrast and a low spatial frequency. The second one is a uniform illumination. The discrepancies in the shrinkage experimented by the material leads to the apparition of patterned stress birefringence revealed trough polarimetry and diffraction measurements.
\end{abstract}

(050.1940) Diffraction and gratings: Diffraction

(160.4890) Materials: Organic Material

(260.1440) Physical optics: Birefringence

(260.5430) Physical optics: Polarization

Nowadays, photopolymers have become true optical materials suitable for the fabrication of diffractive or refractive elements. Beside the possibility of the direct photopatterning of the material surface and refractive index at a microscopic scale, photopolymers simplify and lighten the fabrication of optical elements. Indeed, the patterning is achieved in a one step illumination process which does not require any chemical post-treatment and can be performed at low temperature. The application fields of the photopolymers are principally holography ${ }^{1-3}$ and the microscale optical elements. ${ }^{4-7}$ Moreover, the doping of the photopolymer matrix with push-pull chromophores opened new development to non linear optics ${ }^{8}$ by allowing the spatial control of the quadratic optical non linearity.

In general, the photopolymers are considered as isotropic materials but anisotropy of the refractive index may appear under some specific conditions. Indeed, the birefringence can be obtained by using additives or chemical moieties with defined functions. For example, a photocrosslinkable liquid crystal is able to freeze the mesophase orientation ${ }^{9}$ after polymerization and inherit of permanent birefringence properties. Some specific monomers exhibiting a light polarization dependant photocrosslinking allow the control of the polymeric chain grow direction. These materials are used as liquid crystal alignment layer ${ }^{10}$ for the design of polarization control devices. The birefringence may also appear in non functionalized photopolymers, however in that case they are processed and used in defined conditions. In particular, when sub-wavelength refractive index gratings are built in the medium, the 
birefringence may originate from the so-called form birefringence regime. ${ }^{11}$ One also obtains some birefringence by applying external anisotropic stresses (mechanical, electrical, thermal...). Finally, the diffraction properties of thick gratings ${ }^{13}$ depend also on the polarization state of the incident beam and can then be used to build birefringent elements.

In this letter, we present an original way of inducing spatially distributed birefringence in an acrylate based photopolymer. The material used is free from any optically anisotropic additives. When the photocrosslinking is induced by an anisotropic distribution of the actinic light intensity, the photopolymer undergoes an anisotropic shrinkage that leads to a mechanical stress and consequently to a permanent birefringence. The phenomenon was observed while patterning the material using binary intensity gratings (Ronchi ruling) and studied by characterizing the diffraction properties in the Raman-Nath regime described in the following formalism.

The photocrosslinkable material presented in this paper is prepared in films with a typical thickness of few tens of microns and the grating spacing values explored are large compared to the wavelength. These experimental conditions correspond to the Raman-Nath diffraction regime. Considering a linearly polarized beam at normal incidence, the amplitude of the diffracted intensity in the $l$ order in the Raman-Nath diffraction regime is given by equation $(1):^{12,14}$

$$
A_{i}^{2} J_{l}^{2}\left(\frac{2 \pi}{\lambda} \Delta n_{i} d\right)
$$

where $A_{i}$ is the amplitude of this incident wave linearly polarized in the direction $i, J_{l}$ is the $l^{\text {th }}$ order Bessel function of the first kind, $\lambda$ the wavelength, $d$ the sample thickness and $\Delta n_{i}$ the amplitude of the refractive index modulation in the direction $i$. The polarizations of the different beams are described by their components parallel and perpendicular to the grating wavevector $\vec{K}_{g}$. The electric fields associated to the different beams are given by equation 2 :

$$
\begin{aligned}
& \vec{A}=\|\vec{A}\| \cos \theta \hat{e}_{/ /}+\|\vec{A}\| \sin \theta \hat{e}_{\perp}=A_{/ /} \hat{e}_{/ /}+A_{\perp} \hat{e}_{\perp}, \\
& \vec{A}_{d}^{l}=A_{/ /} \sqrt{\eta_{/ /}^{l}} \hat{e}_{/ /}+A_{\perp} \sqrt{\eta_{\perp}^{l}} \hat{e}_{\perp}
\end{aligned}
$$

where $\vec{A}$ and $\vec{A}_{d}^{l}$ are the electric fields associated respectively to the incident beam and to the diffracted beam at the $l^{\text {th }}$ order, $\theta=\left(\vec{A}, \vec{K}_{g}\right), \hat{e}_{/ /}$and $\hat{e}_{\perp}$ are the unitary vector rescpectively parallel and perpendicular to $\vec{K}_{g}, \eta_{\|}^{l}$ and $\eta_{\perp}^{l}$ the diffraction efficiency for the $l^{\text {th }}$ order in the parallel and perpendicular directions, $A_{/ /}$and $A_{\perp}$ are the corresponding projections of $\vec{A}$. The angle between $\vec{K}_{g}$ and the diffracted beam polarization at $l^{\text {th }}$ order, $\theta_{d}^{l}=\left(\vec{A}_{d}^{l}, \vec{K}_{g}\right)$, is given by relation (3):

$$
\tan \theta_{d}^{l}=\sqrt{\frac{\eta_{\prime \prime}^{l}}{\eta_{\perp}^{l}}} \tan \theta
$$

Equations (2) and (3) show that the intensities of the diffracted beams and moreover the rotation of their polarization depend of the polarization of the incident beam. Here the depolarization due 
to the birefringence is not taken into account as a small birefringence does not induce an important dephasing between the components parallel and perpendicular to $\vec{K}_{g}$ along the propagation in a thin medium.

The material is based on a commercially available trifunctional acrylate monomer the tris[2hydroxy ethyl] isocyanurate triacrylate (SR 368, SARTOMER speciality). This monomer, solid at room temperature $\left(\mathrm{T}_{\mathrm{m}}=56^{\circ} \mathrm{C}\right)$, leads to a high hardness material $\left(\mathrm{T}_{\mathrm{g}}>250^{\circ} \mathrm{C}\right)$ after crosslinking. Its photosensitization in the visible range is achieved by addition of bis(4cylcopentadien-1-yl)-bis[2,6-difluoro-3-(1H-pyrrol-1-yl)-phenyl]titanium (Irgacure 784, Ciba speciality) at a $3 \%$ wt. rate. The Irgacure is directly dissolved in the liquid monomer at $65^{\circ} \mathrm{C}$ without additional solvent and stirred over 12 hours. The mixture is then conditioned in a few tens of microns thick glass cell, filled by capillarity at $65^{\circ} \mathrm{C}$. The shelf lifetime of the samples extends over more than one month. The SR368 tends to crystallize in the cell after a few days, but it can be used after a new heating at $60^{\circ} \mathrm{C}$.

The photopatterning of the material is achieved by imaging the desired pattern in the material. The experimental set-up is shown in Fig. 1. An argon laser provides the actinic light at $514 \mathrm{~nm}$. The object is illuminated by the expanded laser beam. A rotating diffusive disk is inserted at the focus point of the beam expander to homogenize the light distribution by time averaging the speckle. The object is then imaged in the material by an association of two 1.4 numerical aperture $50 \mathrm{~mm}$ camera objectives. The image plane is set in the glass cell placed on a hot-plate. The focus is achieved by adjusting the object position while observing the image quality at the sample plane with a microscope. The objects imaged in the cells are binary intensity gratings with different periods from 3 to 12 lines per millimetre. The sample is placed between crossed polariser and analyser. The variation of the transmitted light intensity related to the bleaching of the photoinitiator is monitored using a photodiode to measure the light taken by a beamsplitter before the analyser. The depolarization due to the induced birefringence is monitored by measuring the light intensity at the exit of the analyzer. The patterning process occurs in two steps. First, the grating is imaged in the material, inducing the photopolymerization of the illuminated areas. In a second step, the grating is removed and the sample is uniformly illuminated. After the patterning process, the observation of the sample with a polarizing microscope reveals an alternation of bright and dark stripes due to the photoinduced birefringence (see Fig. 2).

In bulk polymerization process, the polymerization temperature, the photosensitizer concentration and the illumination conditions greatly affect the final polymerization rate and therefore the material properties. We have studied the evolution of the induced birefringence as a function of the actinic light intensities and the exposure times for the first structured illumination and for the second uniform illumination steps. The patterning protocol leading to the highest observed birefringence is the following. During the whole process, the sample is maintained at $60^{\circ} \mathrm{C}$, slightly above the monomer melting temperature, to insure the fluidity of the mixture and to improve the final polymerization rate. The first illumination is achieved with a power density of $2 \mathrm{~mW} / \mathrm{cm}^{2}$ during 6 minutes. Then, the sample is left 20 minutes in the dark in order to ensure a complete post-polymerization process in the irradiated areas. Finally, the sample is uniformly illuminated with a $0.5 \mathrm{~mW} / \mathrm{cm}^{2}$ power density during 80 minutes and cooled down to room temperature. 
We have characterized the diffraction properties of the photoinduced birefringent gratings using $633 \mathrm{~nm}$ linearly polarized light beam. The grating period was 12 lines per millimetre and the sample thickness was 100 microns. The grating fulfils the Raman-Nath diffraction regime with a Klein-Cook factor ${ }^{15} \mathrm{Q}<0.1$. At a normal incidence angle, the diffraction efficiency of the $+/-1$ order is $1.0 \pm 0.2 \%$ for a light polarization parallel to $\vec{K}_{g}$ and $0.6 \pm 0.2 \%$ for a polarization perpendicular to $\vec{K}_{g}$. No variation of the diffraction angle with polarization was observed. The calculation of the refractive index modulation is achieved considering a sinusoidal grating in the approximation of low modulation amplitude. ${ }^{14}$ The diffraction efficiency data give an index modulation amplitude of $\Delta n_{/ /}=(2.0 \pm 0.2) \cdot 10^{-4}$ in the parallel direction and $\Delta n_{\perp}=(1.5 \pm 0.2) \cdot 10^{-4}$ in the perpendicular direction. When positioning the sample between two polarizers, the extinction of the transmitted beam and of the diffracted beam are observed at different orientations of the output polarizer. For $+/-1$ order diffracted order, the largest rotation of the beam polarization was $\theta_{d}^{l}-\theta=22^{\circ}$, obtained for an incident beam polarization angle $\theta$ around $45^{\circ}$ (see Fig. 3). As expected, the minimum orientation difference was observed for a polarization of the incident beam along the two directions $\hat{e}_{/ /}$and $\hat{e}_{\perp}$. All these results are consistent with the calculations presented above. The model does not take into account a possible dephasing between the two orthogonal polarization components. This agrees with the experimental results, as no sensitive depolarisation of the transmitted or the diffracted beam is observed. Indeed, if one considers a sample with a uniform $5 \cdot 10^{-5}$ birefringence and a 100 microns thickness, at normal incidence, the dephasing between the parallel and perpendicular components is about $2^{\circ}$ only and therefore can be neglected in the description of the diffraction.

A similar polarization effect has already been demonstrated in acousto-optics experiments ${ }^{16}$ for crystals under mechanical stress. Nevertheless, with photopolymers, it is possible to record much more complex birefringence distributions and do this more easily than using acousto-optic effect.

If the induced birefringence is negligible in the diffraction description, the induced depolarization is high enough to be observed. Advantage is taken of this depolarization to monitor the establishment of the birefringence. For this purpose, the patterning is achieved with a sample placed between crossed polarizers (see Fig. 1). The grating direction is oriented at $45^{\circ}$ from the incident light polarization direction. The light intensity transmitted through the sample is measured before and after the analyzer in order to separate the contribution of the photoinitiator bleaching from the birefringence one. The depolarization of the transmitted light is already observed at the first step of the photopatterning process. This tendency remains in the second step until its saturation at the end of the uniform illumination. We interpret the origin of the photoinduced birefringence as the effect of stored mechanical constrains due to the non uniform shrinkage. Indeed, it is well known that the polymerization of bulk acrylate monomer based materials is accompanied by a matter densification inducing an increase of the refractive index and the shrinkage of the medium. These stresses modify the refractive index ellipsoid via elasto-optic effect. It is worthy to note that this phenomenon is only observed in high hardness material where the induced strains are not able to relax at short time scale. The photopatterning of softer materials based on other monomers as pentaerytritol triacrylate or even on plasticized SR368 doesn't induce any observable birefringence. The observation of a depolarization at the first polymerization step indicates that the anisotropic light distribution corresponding to an inhomogeneous polymerization leads to stress distribution. This phenomenon is explained considering the variation of the shrinkage with the direction leading to anisotropic strains. 
Therefore, by knowing the elasto-optic coefficient of the material, it would be possible to evaluate the strains induced in the materials. For a variation of the sample thickness (between 40 and $200 \mu \mathrm{m}$ ) and of the grating period (between 3 and 12 lines $/ \mathrm{mm}$ ), the diffraction efficiency remains around $1 \%$ and the birefringence around a few $10^{-5}$. These variations are low and the influence of the experimental conditions cannot be distinguished from the effect of samples variability. This agrees with the elasto-optics origin as the effect depends on the deformation rate and not with the absolute deformation. But, the spatial resolution of the imaging setup and of the material itself would limit the observation of birefringence at very low grating spacing. Nevertheless, the examination with the polarizing microscope shows that 120 lines $/ \mathrm{mm}$ grating still presents some birefringence which gives hope to go to the sub-micron scale after optimization of the material, of the set-up (spatial resolution, depth of field) and of the process. As mentioned before, the photopolymerization conditions (temperature, light intensity, exposure time) have a sensitive influence. The conditions used here are comparable to these leading to a high polymerization rate of the final product and therefore to a high hardness material. Finally, the lifetime of the induced birefringence distribution appears to be very long. No modification of the samples properties was observed over more than six months for samples stored at room temperature.

We have demonstrated that a permanent distribution of birefringence may be induced by a photopatterning of high hardness photocrosslinkable materials. The inscription of diffraction gratings, operating in the Raman-Nath regime, was achieved and we observed a rotation of the polarization direction between the incident and the diffracted beams. The birefringence was attributed to elasto-optic effect in the material where the photopolymerization using an anisotropic light intensity distribution has induced anisotropic strains in the material.

The development of this topic requires now the development a new photocrosslinkable material with improved birefringence properties. Among the different solutions arising, the most promising one is the use of photocrosslinkable sol-gel matrix. Indeed, they present a better hardness compare to organics systems. The possibility of using different metal alkoxy should also provide a better control of the birefringence.

The mastering of the birefringent grating would allow the control of the diffraction properties by the control of the polarization of the incident beam. Here only linear gratings have been considered. It is possible to use more complicated illumination patterns (e.g. checkers board, concentric circles) leading to very complex birefringence distributions that may be used to control the polarization distribution in a light beam profile. Finally, the control of the birefringence at a microscopic scale will recover a great interest in the development and the optimization of diffractive optics elements. ${ }^{17,18}$

\section{Acknowledgement}

The authors acknowledge the Région Alsace and the INTERREG III / Rhenaphotonics program for their financial supports. 


\section{References:}

1. L. Dhar, K. Curtis, M. Tackitt, M. Schilling, S. Campbell, W. Wilson, A. Hill, C. Boyd, N. Levinos, A. Harris, "Holographic storage of multiple high-capacity digital data pages in thick photopolymer systems", Opt. Lett. 23, 1710-1712 (1998).

2. J. Hewett, "Holographic drives set for long-awaited debut", Opto \& Laser Europe 119, 15-17 (2004).

3. J. Guo, M.R. Gleeson, J.T. Sheridan, "A review of the optimisation of photopolymer materials for holographic data storage”, Phys. Res. Int. 2012, 803439 (2012).

4. C. Croutxe-Barghorn, O. Soppera, D.J. Lougnot, "Fabrication of microlenses by direct photo-induced crosslinking polymerization", Appl. Surf. Sci. 168, 89-91 (2000).

5. X. Zeng, J. Plain, S. Jradi, C. Darraud, F. Louradour, R. Bachelot, P. Royer, "Integration of polymer microlens array at fiber bundle extremity by photopolymerization", Opt. Express 19, 4805-4814 (2011).

6. V. Pavelyev, V. Osipov, D. Kachalov, B. Chichkov, "Diffractive optical elements with radial fourlevel microrelief fabricated by two-photon polymerization”, Opt. Comm. 286, 368-371 (2013).

7. J. Neumann, K. S. Wieking, D. Kip, "Direct laser writing of surface reliefs in dry, self-developing photopolymer films", Applied Optics 38, 5418-5421 (1999).

8. F. Gillot, L. Mager, K.D. Dorkenoo, S. Mery, C. Carre, A. Fort, "Photo-patterning of the quadratic optical properties of doped photopolymers", Chem. Phys. Lett. 379, 203-208 (2003).

9. H. Ono, A. Emoto, N. Kawatsuki, E. Uchida, M. Kuwabara, "New fabrication method for anisotropic gratings formed in photocrosslinkable polymer liquid crystals", Appl. Phys. A 79, 1725-1727 (2004).

10. M. Schadt, H. Seiberle, A. Schuster, "Optical patterning of multi-domain liquid-crystal display with wide viewing angles", Nature 381, 212-215 (1996).

11. C. Yang, P. Yeh, "Form birefringence of volume gratings in photopolymers", Appl. Phys. Lett., 69, 3468 (1996).

12. M. Born, E.Wolf, Principles of optics (Pergamon Press, 1993).

13. H. Kogelnick, "Coupled wave theory for thick hologram gratings", Bell Syst. Techn. Journal, 48, 2909-2947 (1969).

14. J.W. Goodman, Introduction à l'optique de Fourier et à l'holographie (Masson \& Cie, Paris, 1972).

15. W. R. Klein, B. D. Cook, "Unified Approach to Ultrasonic Light Diffraction", Trans. Sonics Ultrason. SU-14, 123-134 (1967).

16. E. Blomme, G. Gondek, T. Katkowski, P. Kwiek, A. Sliwinski, O. Leroy, "On the polarization of light diffracted by ultrasound", Ultrasonics 38, 575-580 (2000).

17. H. Lajunen, J. Tervo, J. Turunen, "High efficiency broadband diffractive elements based on polarization gratings", Opt. Lett. 29, 803-805 (2004).

18. M. Fratz, D.M. Giel, P. Fischer, "Digital polarization holograms with defined magnitude and orientation of each pixel's birefringence", Opt. Lett. 34, 1270-1272 (2009). 


\section{Figure captions}

Figure 1: Experimental setup. (M) microscope objective, (R) rotating diffusive disk, (C) collimating lens, $(\mathrm{G})$ binary grating, $(\mathrm{O})$ Nikon AF NIKKOR $50 \mathrm{~mm}$ camera objectives, $\left(\mathrm{P}_{1}\right)$ polarizer, $\left(\mathrm{P}_{2}\right)$ analyzer, $(\mathrm{H})$ hot plate, $(\mathrm{S})$ sample, $(\mathrm{B})$ beamsplitter, $\left(\mathrm{D}_{1}\right)$ photodiode for the monitoring of the variation of the transmitted intensity due to the photoinitiator bleaching, (D2) photodiode for the monitoring of the variation of the transmitted intensity due to the depolarization of light.

Figure 2: Image of the photopatterned sample using a polarizing microscope. (a) Incident polarization parallel to the grating wavevector, (b) incident polarization at $45^{\circ}$ from the grating wavevector, (c) incident polarization orthogonal to the grating wavevector.

Figure 3: Polar plot of the intensity versus the exit polarizer orientation for a polarization of the incident beam at $45^{\circ}$ from the grating wavevector. Solid squares: transmitted beam, hollow squares: first order diffracted beam. The curves correspond to the square cosine fit. The transmitted beam shows no depolarization. The $1^{\text {st }}$ order diffracted beam is linearly polarized and its polarization is rotated with a $22^{\circ}$ angle compared to the transmitted beam. 


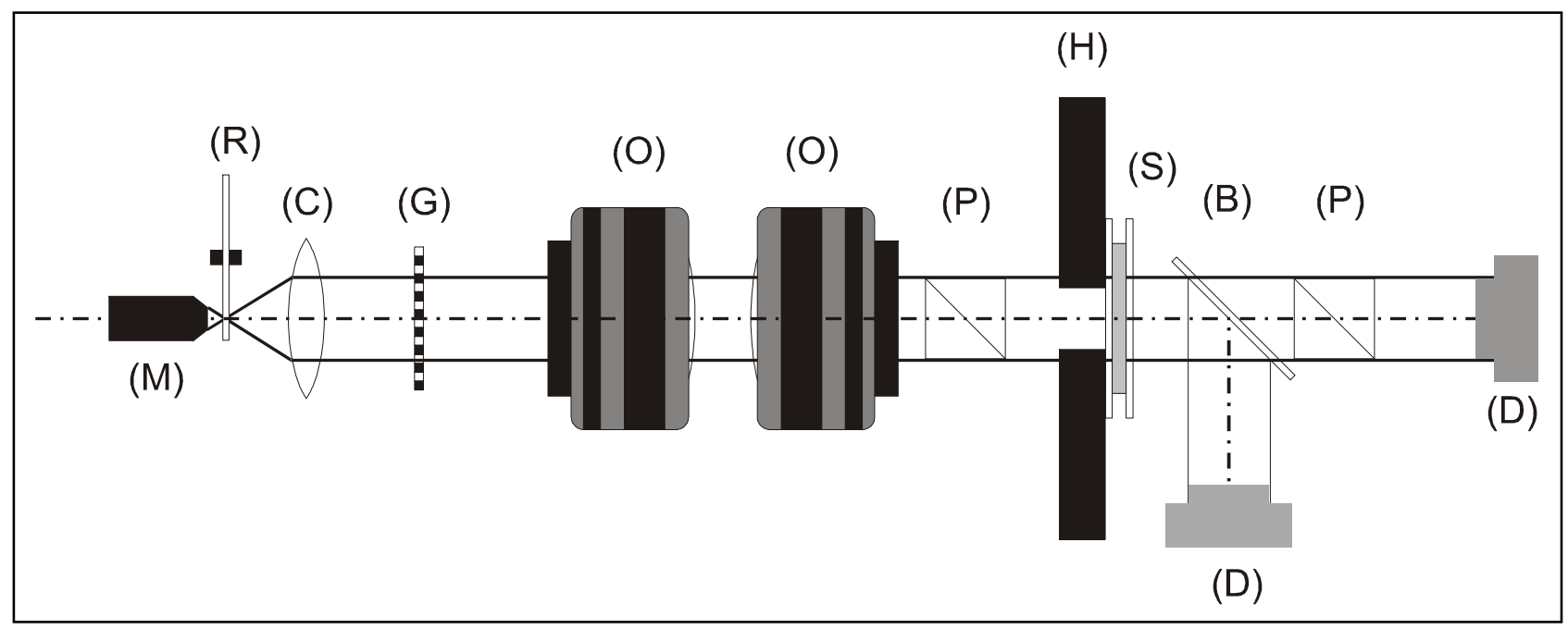

Figure 1 


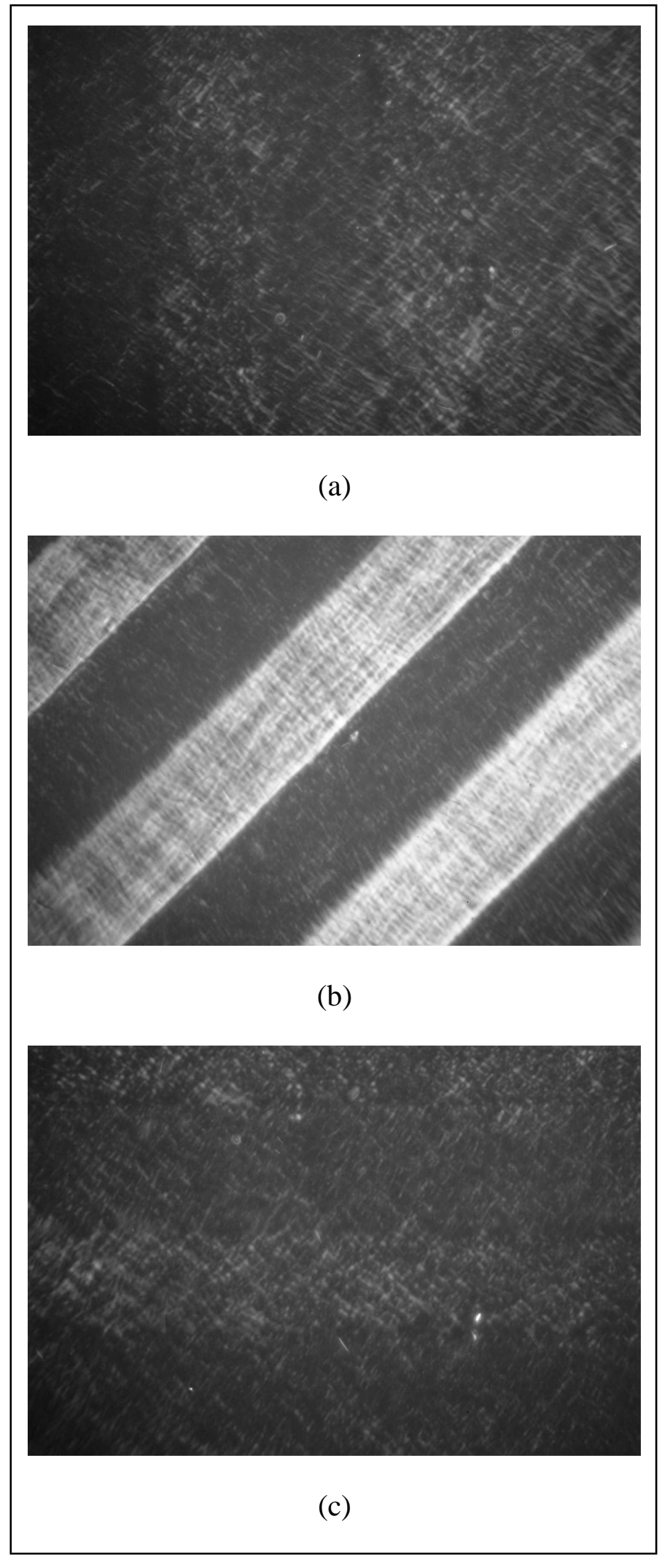

Figure 2 


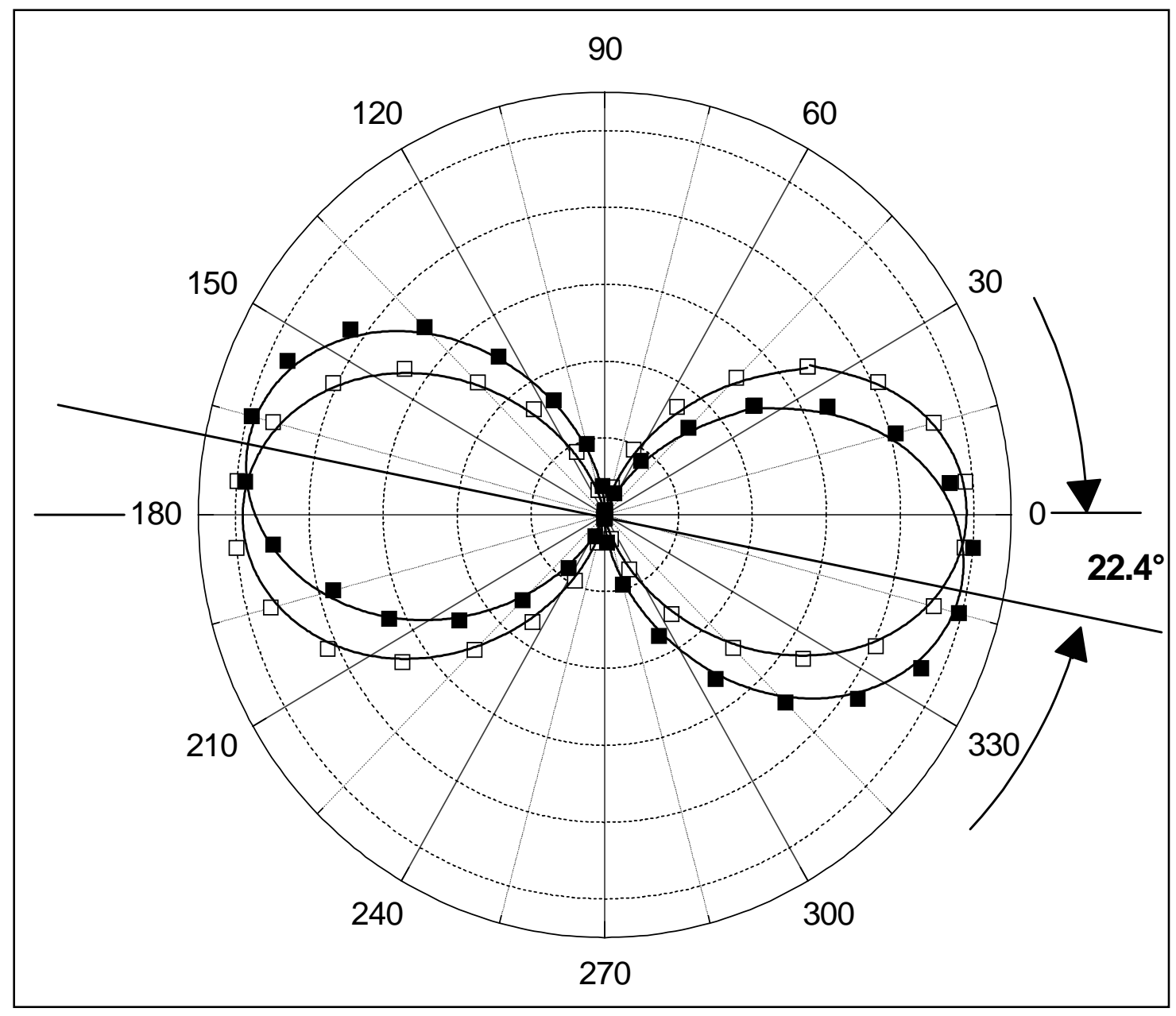

Figure 3 